\title{
How does the quantum structure of electromagnetic waves describe quantum redshift?
}

Bahram Kalhor ( $\nabla$ kalhor_bahram@yahoo.com )

Islamic Azad University , Karaj https://orcid.org/0000-0002-6700-6269

\section{Farzaneh Mehrparvar}

Azad University

\section{Behnam Kalhor}

Karaj Branch, Azad University

\section{Article}

Keywords: quantum structure, electromagnetic waves, quantum redshift, z parameter

Posted Date: October 12th, 2020

DOI: https://doi.org/10.21203/rs.3.rs-87775/v1

License: (1) This work is licensed under a Creative Commons Attribution 4.0 International License.

Read Full License 


\title{
How does the quantum structure of electromagnetic waves describe quantum redshift?
}

\begin{abstract}
The paper introduces quantum redshift. By using the quantum structure of the electromagnetic waves, we can describe the redshift. Losing the quanta masses along the traveling in the space is the reason of the decreasing the frequency of the electromagnetic waves. Recursive quantum redshift predict distance of the objects by calculating the $\mathrm{z}$ parameter of the waves since they have emitted. Non-recursive quantum redshift is a fast and good approximation of the recursive quantum redshift. The distances in the quantum redshift is less than the distances in the accelerated expansion space theory. The paper provides $\mathrm{z}$ parameter of distances between zero and 12 billion light years.
\end{abstract}

\section{Introduction}

Cosmic redshift or shift in spectral lines is a powerful tool for calculating the distance of the objects in the universe. It belongs to the electromagnetic waves area and usually happens when the wavelength of a wave decrease by traveling in the space. The measurement parameter of the cosmic redshift is $\mathrm{z}$. The value of the $\mathrm{z}$ usually is a positive number, and more distance is equal to the greater $\mathrm{z}$ value. In some cases, the value of the $\mathrm{z}$ is negative, and we have Blueshift.

Although the redshift is known by name of the Edwin Hubble [1], Vesto Slipher (1875-1969) was the first astronomer who measured it [2]. Also, the redshift of the cosmic waves has measured by Carl W. Wirtz (1922) and the Swede Knut Lundmark (in 1924) [3,4].

There are many theories for describing the behavior of the wave in the space and increasing its wavelength. Doppler effect is the most similar theory that has been used for investigating the cosmic redshift [5-9]. In the Doppler effect, changing the wavelength is due to changing of the distance between emitter and observer during the traveling of the wave between them. In 1848 Hippolyte Fizeau proposed that cosmic redshift is like the Doppler effect. In 1968 William Huggins measured the velocity of the stars by using the Doppler effect formula. According to this method, objects that come toward us have the Blueshift, while objects that move away have the redshift.

Using spectral lines and the Doppler effect method showed that the speed of some stars and galaxies should be more than the speed of light. This issue disagreed with Einstein's special relativity [13-16]. Hence, the expanding universe theory is proposed. In the expanding space theory, the distance between two objects in the space will be increased along the time even they do not move. In the expanding space theory, the reason for the redshift of the waves is expanding the space. Also, by increasing the distance between the objects their expansion rate will be increased. 
If we accept the expansion of the space, we should find dark energy that makes this expansion. The expanding space theory has two problems. The dark energy is not discovered yet, and not possible to build a realistic model of the universe on modes of unrestrained expansion [17].

The gravitational redshift is another theory that tries to describe the spectral displacement by using general relativity [18-22]. Although there is a significant redshift for massive objects, it is a weak effect for non-massive stars.

The purpose of this work is to represent recursive quantum redshift and non-recursive quantum redshift for measuring the distance of the objects. Concepts of the quanta mass [23] and quantum structure of the electromagnetic waves [24] are the main parts of this theory. In the quantum redshift, regardless of the frequency of the wave, each period of the wave conclude equal number of the quanta masses. The number of the quanta masses in each period will be decreased by traveling in the space, hence the energy of the wave will be decreased.

The quantum redshift disagreed with the accelerated expansion of the space; hence the results of the quantum redshift show that the real distances of the objects are less than the distances that have been obtained in the expanding space theory.

\section{Recursive quantum redshift}

The frequency of the wave is equal to the total number of the $\mathrm{k}$ boxes that carry in a second. On the other hand, while $\mathrm{k}$ boxes move in a vacuum, in each second, one quantum of the mass will be decreased from each $\mathrm{k}$ box. Hence, in each second, the total number of the decreased quanta masses that have distributed in 299792458 meters is equal to the frequency of the wave at that time multiplied by the $\mathrm{p}$ or $f_{t_{n}} * p$ where $\mathrm{p}$ is the number of the quanta masses that will be decreased in each second from each $\mathrm{k}$ box and $f_{t_{n}}$ is the frequency of the wave at the start of each second or total number of the $\mathrm{k}$ boxes that wave carry in a second.

If result of the $f_{t_{n}} * p$ be less than the $\mathrm{q}$, the frequency will not be decreased and this operation will be continued $t_{n}$ seconds until the sum of the lost quantum of the masses for all the $\mathrm{k}$ boxes reaches to q. After passing $t_{n}$ seconds wave has concluded $f_{t_{n}} \mathrm{k}$ boxes that all of them are not the full field, hence some of them will be destroyed and send their remain quanta masses to others, this operation will be decreased frequency of the wave. The amount of the $t_{n}$ is given by:

$$
t_{n}=\left\lceil\frac{q}{f_{t_{n}}}\right\rceil
$$

Where the $t_{n}$ is the counts of seconds that $\mathrm{k}$ boxes lose their quanta masses until the sum of the lost quantum masses reaches the capacity of the one $k$ box (q). If $f_{t_{n}}>q$ then $t_{n}=1$.

The total number of the decreased quanta masses in $t_{n}$ second is given by:

$$
\begin{gathered}
\mathrm{S}_{t_{n}}=t_{n} * f_{t_{n}} * p+R_{t_{n-1}} \\
R_{t_{0}}=0
\end{gathered}
$$


Where $S_{t_{n}}$ is the total number of the lost quanta masses in the $t_{n}$ seconds and $R_{t_{n-1}}$ is the remain number of division $\frac{\mathrm{s}_{t_{n}}}{q}$ in the previous step $\left(0 \leq R_{t_{n-1}}<q\right)$.

After passing $t_{n}$ seconds and reaching the number of the lost quanta masses $\left(\mathrm{S}_{t_{n}}\right)$ to greater than the capacity of the one $\mathrm{k}$ box (q), $\mathrm{k}$ boxes will be reconstructed and the wave will be lost some $\mathrm{k}$ boxes by sharing remain quanta masses of lost $\mathrm{k}$ boxes for fulling fields remain $\mathrm{k}$ boxes. Hence, frequency will be decreased. Equation is given by:

$$
f_{l_{n}}=\left[\frac{\mathrm{S}_{t_{n}}}{q}\right]
$$

Where $f_{l_{n}}$ is the amount of the frequency that will be decreased.

Table.1 illustrates the parameters of the quantum redshift in each step.

Table.1: Parameters of the Recursive quantum redshift in each step.

\begin{tabular}{|c|c|c|c|c|c|}
\hline$f_{t_{n}}$ & $t_{n}=\left[\frac{q}{f_{t_{n}}}\right]$ & $\begin{array}{c}\mathrm{S}_{t_{n}}=R_{t_{n-1}}+t_{n} * f_{t_{n}} * p \\
\left(R_{t_{0}}=0\right)\end{array}$ & $f_{l_{n}}$ & $R_{t_{n}}=\mathrm{S}_{t_{n}}-f_{l_{n}} * q$ & $f_{t_{n+1}}=f_{t_{n}}-f_{l_{n}}$ \\
\hline$f_{t_{1}}=f_{\text {emit }}$ & {$\left[\frac{q}{f_{t_{1}}}\right]$} & $\mathrm{S}_{t_{1}}=R_{t_{0}}+t_{1} * f_{t_{1}} * p$ & {$\left[\frac{S_{t_{1}}}{q}\right]$} & $R_{t_{1}}=\mathrm{S}_{t_{1}}-f_{l_{1}} * q$ & $f_{t_{2}}=f_{t_{1}}-\left[\frac{S_{t_{1}}}{q}\right]$ \\
\hline$f_{t_{2}}=f_{t_{1}}-\left[\frac{S_{t_{1}}}{q}\right]$ & {$\left[\frac{q}{f_{t_{2}}}\right]$} & $\mathrm{S}_{t_{2}}=R_{t_{1}}+t_{2} * f_{t_{2}} * p$ & {$\left[\frac{S_{t_{2}}}{q}\right]$} & $R_{t_{2}}=S_{t_{2}}-f_{l_{2}} * q$ & $f_{t_{3}}=f_{t_{2}}-\left[\frac{S_{t_{2}}}{q}\right]$ \\
\hline$f_{t_{3}}=f_{t_{2}}-\left[\frac{S_{t_{2}}}{q}\right]$ & {$\left[\frac{q}{f_{t_{3}}}\right]$} & $\mathrm{S}_{t_{3}}=R_{t_{2}}+t_{3} * f_{t_{3}} * p$ & {$\left[\frac{S_{t_{3}}}{q}\right]$} & $R_{t_{3}}=S_{t_{3}}-f_{l_{3}} * q$ & $f_{t_{4}}=f_{t_{3}}-\left[\frac{S_{t_{3}}}{q}\right]$ \\
\hline$f_{t_{n}}=f_{t_{n-1}}-\left[\frac{S_{t_{n-1}}}{q}\right]$ & {$\left[\frac{q}{f_{t_{n}}}\right]$} & $\mathrm{S}_{t_{n}}=R_{t_{n-1}}+t_{n} * f_{t_{n}} * p$ & {$\left[\frac{S_{t_{n}}}{q}\right]$} & $R_{t_{n}}=S_{t_{n}}-f_{l_{n}} * q$ & $f_{t_{n+1}}=f_{t_{n}}-\left[\frac{S_{t_{n}}}{q}\right]$ \\
\hline
\end{tabular}

The results of the equation $\frac{\mathrm{s}_{n}}{q}$ is not an integer number, hence a few numbers of $\mathrm{k}$ boxes will be remained, and we should consider them in the next operation, hence:

$$
R_{t_{n}}=\mathrm{S}_{t_{n}}-f_{l_{n}} * q
$$


on the other hand,

$$
f_{t_{n+1}}=f_{t_{n}}-f_{l_{n}}
$$

where

$$
f_{\text {emit }}=f_{t_{1}}
$$

and

$$
f_{o b s}=f_{t_{n+1}}
$$

in the quantum redshift

$$
f_{t_{n+1}}=f_{t_{n}}-\left[\frac{s_{t_{n}}}{q}\right]
$$

also

$$
f_{t_{n}}=f_{t_{n-1}}-\left[\frac{\mathrm{S}_{t_{n-1}}}{q}\right]
$$

and

$$
f_{t_{n-1}}=f_{t_{n-2}}-\left[\frac{\mathrm{S}_{t_{n-2}}}{q}\right]
$$

hence

$$
f_{t_{n+1}}=f_{t_{n-1}}-\left[\frac{S_{t_{n-1}}}{q}\right]-\left[\frac{S_{t_{n}}}{q}\right]
$$

so

$$
f_{t_{n+1}}=f_{t_{n-2}}-\left[\frac{S_{t_{n-2}}}{q}\right]-\left[\frac{S_{t_{n-1}}}{q}\right]-\left[\frac{S_{t_{n}}}{q}\right]
$$

or

$$
f_{t_{n+1}}=f_{t_{1}}-\sum_{k=0}^{n-1}\left[\frac{S_{t_{n-k}}}{q}\right]
$$

using (6) and (7)

$$
f_{o b s}=f_{e m i t}-\sum_{k=0}^{n-1}\left[\frac{S_{t_{n-k}}}{q}\right]
$$

on the other hand,

$$
z=\frac{f_{\text {emit }}-f_{o b s}}{f_{o b s}}
$$

using (11) 


$$
z=\frac{f_{\text {emit }}-f_{\text {emit }}-\sum_{k=0}^{n-1}\left[\frac{\mathrm{S}_{t_{n-k}}}{q}\right]}{f_{\text {emit }}-\sum_{k=0}^{n-1}\left[\frac{\mathrm{S}_{t_{n-k}}}{q}\right]}
$$

so

$$
z+1=\frac{f_{\text {emit }}}{f_{\text {emit }}-\sum_{k=0}^{n-1}\left[\frac{S_{t_{n-k}}}{q}\right]}
$$

The equation (11) provides the amount of the frequency in the next step based on the frequency of the previous step. Also, the equation (1) provides the time for decreasing frequency in each step. In the quantum redshift for calculating the distance of a remote galaxy we use the total time of traveling the wave between the galaxy and the observer, the equation is given by:

$$
\mathrm{t}=\sum_{i=1}^{n} t_{i}
$$

so

$$
d=c * \sum_{i=1}^{n} t_{i}
$$

In the equations (13) and (14) amount of the parameter $\mathrm{n}$ is not specified at the first. These equations represent recursive procedure; hence we need a computer program that according to the $f_{\text {obs }}$ step by step calculate previous frequencies and time of that step until the frequency reaches to the $f_{\text {emit }}$.

\section{Approximating recursive quantum redshift to non-recursive quantum redshift}

Although environmental parameters such a temperature, have an impact on the $\mathrm{p}$, in a normal space we can use it as a constant value. Also, the value of the $q$ is invariant, On the other hand, in the equation (4), the amount of the $R_{t_{n}}$ is too small and we can omit it. A simple relationship between $f_{t+1}$ and $f_{t}$ in each second is given by:

$$
f_{t+1}=f_{t}-f_{t} \times \frac{p}{q}
$$

where $\mathrm{p}=1, \mathrm{q}=89875518173474223$, and $\frac{p}{q}=1.112650052342217352357 \mathrm{E}-17$

Hence, $\mathrm{t}$ seconds after emitting, $f_{\text {obs }}$ would be obtained depend on $f_{\text {emit }}$ by this equation:

$$
f_{\text {obs }}=f_{\text {emit }}\left(1-\frac{p}{q}\right)^{t}
$$

The equation (16) is a non-recursive quantum redshift.Table. 2 shows the changes of the frequency in consequence seconds, respectively. 
Table.2: Parameters of the non-Recursive quantum redshift in each step.

\begin{tabular}{|c|c|c|c|c|}
\hline Time & Frequency & $f_{l_{t}}$ & $f_{o b s}=f_{\text {emit }}-f_{l_{t}}$ & $f_{o b s}$ \\
\hline 1 & $f_{\text {emit }}$ & $f_{\text {emit }} \times \frac{p}{q}$ & $f_{\text {emit }}-f_{\text {emit }} \times \frac{p}{q}$ & $f_{\text {emit }}\left(1-\frac{p}{q}\right)$ \\
\hline 2 & $f_{\text {emit }}\left(1-\frac{p}{q}\right)$ & $f_{\text {emit }}\left(1-\frac{p}{q}\right) \times \frac{p}{q}$ & $\begin{array}{l}f_{\text {emit }}\left(1-\frac{p}{q}\right)- \\
f_{\text {emit }}\left(1-\frac{p}{q}\right) \times \frac{p}{q}\end{array}$ & $\begin{array}{c}f_{\text {emit }}\left(1-\frac{p}{q}\right)\left(1-\frac{p}{q}\right) \\
=f_{\text {emit }}\left(1-\frac{p}{q}\right)^{2}\end{array}$ \\
\hline 3 & $f_{\text {emit }}\left(1-\frac{p}{q}\right)^{2}$ & $f_{\text {emit }}\left(1-\frac{p}{q}\right)^{2} \times \frac{p}{q}$ & $\begin{array}{c}f_{\text {emit }}\left(1-\frac{p}{q}\right)^{2}- \\
f_{\text {emit }}\left(1-\frac{p}{q}\right)^{2} \times \frac{p}{q}\end{array}$ & $\begin{array}{c}f_{\text {emit }}\left(1-\frac{p}{q}\right)^{2} \times\left(1-\frac{p}{q}\right) \\
=f_{\text {emit }}\left(1-\frac{p}{q}\right)^{3}\end{array}$ \\
\hline $\mathrm{t}$ & $f_{\text {emit }}\left(1-\frac{p}{q}\right)^{t-1}$ & $f_{\text {emit }}\left(1-\frac{p}{q}\right)^{t-1} \times \frac{p}{q}$ & $\begin{array}{l}f_{\text {emit }}\left(1-\frac{p}{q}\right)^{t-1}- \\
f_{\text {emit }}\left(1-\frac{p}{q}\right)^{t-1} \times \frac{p}{q}\end{array}$ & $f_{\text {emit }}\left(1-\frac{p}{q}\right)^{t}$ \\
\hline
\end{tabular}

\section{Calculating time by using frequency}

By using the equation of the non-recursive quantum redshift and according to the definition of the $\mathrm{z}$ we can calculate the time between the emitter and observer.

$$
z=\frac{f_{e m i t}-f_{o b s}}{f_{o b s}}
$$

using (16)

$$
Z=\frac{f_{\text {emit }}-f_{\text {emit }}\left(1-\frac{p}{q}\right)^{t}}{f_{\text {emit }}\left(1-\frac{p}{q}\right)^{t}}
$$

so

$$
Z=\frac{1-\left(1-\frac{p}{q}\right)^{t}}{\left(1-\frac{p}{q}\right)^{t}}
$$

or

$$
z+1=\frac{1}{\left(1-\frac{p}{q}\right)^{t}}
$$

$\left(1-\frac{p}{q}\right)$ is a constant value, hence 


$$
z+1=\frac{1}{\beta^{t}}
$$

where

$$
\beta=1-\frac{p}{q}
$$

so

$$
t=\log _{\beta}\left(\frac{1}{Z+1}\right)
$$

On the other hand, $\mathrm{p}=1$ and $\mathrm{q}=89875518173474223$, hence

$$
\beta=0.9999999999999999888735
$$

\section{Calculating distance by using frequency}

The equation (16) represents the relationship between the $f_{\text {emit }}$ and $f_{\text {obs }}$.

$$
f_{\text {obs }}=f_{\text {emit }}\left(1-\frac{p}{q}\right)^{t}
$$

on the other hand,

$$
t=\frac{d}{c}
$$

hence

$$
f_{\text {obs }}=f_{\text {emit }}\left(1-\frac{p}{q}\right)^{\frac{d}{c}}
$$

so

$$
\frac{f_{\text {obs }}}{f_{\text {emit }}}=\left(1-\frac{p}{q}\right)^{\frac{d}{c}}
$$

or

$$
\frac{d}{c}=\log _{\left(1-\frac{p}{q}\right)}\left(\frac{f_{o b s}}{f_{e m i t}}\right)
$$

using (22)

$$
d=c \times \log _{\beta}\left(\frac{f_{o b s}}{f_{\text {emit }}}\right)
$$

\section{Calculating distance by using $z$}

Using equations (20) and (25) 


$$
z+1=\frac{1}{\left(1-\frac{p}{q}\right)^{\frac{d}{c}}}
$$

or

$$
z+1=\left(1-\frac{p}{q}\right)^{-\frac{d}{c}}
$$

so

$$
-\frac{d}{c}=\log _{\left(1-\frac{p}{q}\right)}(z+1)
$$

using (22)

$$
d=-c * \log _{\beta}(z+1)
$$

\section{Discussion}

In the real world, scientists obtain the parameter $\mathrm{z}$ of the objects in the space and calculate their distance to the observer. The equation (14) provides a recursive quantum redshift method for calculating the distance of the objects while the equation (30) represents a non-recursive quantum redshift method. The advantage of the non-recursive quantum redshift method is its higher speed of the calculation. For calculating the distance of the object by using the equation (14) we need a computer program and fast computer, but equation (30) is a simple equation that could be calculated by a professional calculator. The only restriction of the equation (30) is the value of the $\beta$, it is too close to the 1 , and for calculating $\log _{\beta}$ we need a calculator that supports this kind of calculation. In this paper, we have used an online calculator from this internet address https://keisan.casio.com/calculator.

However, we should compare the results of both methods to ensure that the results of the nonrecursive quantum redshift method are reliable. For this reason, we wrote a program and calculate the parameter $\mathrm{z}$ for distances between zero to almost 8 billion light-years. This range of distances covers $\mathrm{z}$ parameters between zero and 12. In the table.3 the columns (1) and (2) represent the relation between the special distances and their $\mathrm{z}$ value in the recursive quantum redshift, respectively. We should consider that result in the quantum redshift is not in agreement with the accelerated expansion universe theory, hence the distances that would be obtained from each $\mathrm{z}$ by the quantum redshift would be less than the distances that has been calculated by the accelerated expansion universe method.

In the next step, we used all z parameters in the column (2) for calculating the distances of the objects in equation (30). The results have been shown in the column (3). The difference between

the two methods is too small, and less than $1 * 10^{-5}$ percent, hence results of the non-recursive quantum method are reliable. 
Table.3: Comparing distances in the Recursive quantum redshift and non-Recursive quantum redshift.

\section{Quantu0m redshift}

\begin{tabular}{|c|c|c|}
\hline \begin{tabular}{|c|} 
Recursive \\
(Light Year)
\end{tabular} & $\mathbf{Z}$ & $\begin{array}{c}\text { non-Recursive } \\
\text { (Light Year) }\end{array}$ \\
\hline 0 & 0 & 0.00 \\
\hline 200000000 & 7.27496578431443E-02 & $199,999,996.17$ \\
\hline 400000000 & 0.150791769911269 & $399,999,847.59$ \\
\hline 600000000 & 0.234511509968063 & $599,999,918.85$ \\
\hline 800000000 & 0.324321765161994 & $799,999,840.27$ \\
\hline 1000000000 & 0.420665803908163 & $1,000,000,003.75$ \\
\hline 1200000000 & 0.524018677229894 & $1,199,999,854.50$ \\
\hline 1400000000 & 0.634890525686787 & $1,399,999,870.08$ \\
\hline 1600000000 & 0.753828278288702 & $1,599,999,908.87$ \\
\hline 1800000000 & 0.881418650808816 & $1,799,999,852.61$ \\
\hline 2000000000 & 1.01829126919994 & $1,999,999,926.79$ \\
\hline 2200000000 & 1.16512135640523 & $2,200,000,038.65$ \\
\hline 2400000000 & 1.32263320410299 & $2,400,000,046.88$ \\
\hline 2600000000 & 1.4916040729006 & $2,600,000,154.96$ \\
\hline 2800000000 & 1.67286727326276 & $2,799,999,998.31$ \\
\hline 3000000000 & 1.86731773894792 & $3,000,000,278.65$ \\
\hline 3200000000 & 2.07591394054181 & $3,200,000,105.53$ \\
\hline 3400000000 & & $3,399,999,972.96$ \\
\hline 3600000000 & 081 & $0,288.70$ \\
\hline 3800000000 & 2.79725145446597 & $3,800,000,242.78$ \\
\hline 4000000000 & 3.07350020087448 & $4,000,000,240.60$ \\
\hline 4200000000 & 3.36984631250519 & $4,200,000,475.17$ \\
\hline 4400000000 & 3.68775041010491 & $4,400,000,030.00$ \\
\hline 4600000000 & 4.0287834995 & 4,600, \\
\hline 4800000000 & 4.39462526741326 & $4,800,000,234.50$ \\
\hline 5000000000 & 4.78708308587525 & $5,000,000,563.39$ \\
\hline 5200000000 & & $0,164.35$ \\
\hline 5400000000 & 5.65972667649254 & $5,400,000,021.60$ \\
\hline 5600000000 & 6.14421900960585 & $5,599,999,816.89$ \\
\hline 5800000000 & 6.66395841536164 & $5,799,999,782.31$ \\
\hline 6000000000 & 7.22150797871657 & $5,999,999,505.14$ \\
\hline 6200000000 & 7.8196205688986 & $6,199,999,726.63$ \\
\hline 6400000000 & 8.46124702783795 & $6,400,000,348.99$ \\
\hline 6600000000 & 9.14954723504131 & $6,599,999,706.28$ \\
\hline 6800000000 & 9.88792379542136 & $6,799,999,825.86$ \\
\hline 7000000000 & 10.6800171696252 & $6,999,999,978.93$ \\
\hline 7200000000 & 11.529738551343 & $7,200,000,913.62$ \\
\hline 7400000000 & 12.4412667049743 & $7,399,999,630.26$ \\
\hline
\end{tabular}

Fig.1 illustrates the relationship between distances and their $\mathrm{z}$ parameters in the quantum redshift theory. By increasing the distance, the $\mathrm{z}$ will be increased more. Meanwhile, this graph shows that the percent of the increase in the $\mathrm{z}$ parameter is more than the increasing percentage of the distance. This agrees with the real data of the universe.

Fig.1: distances and their z parameters in the quantum redshift

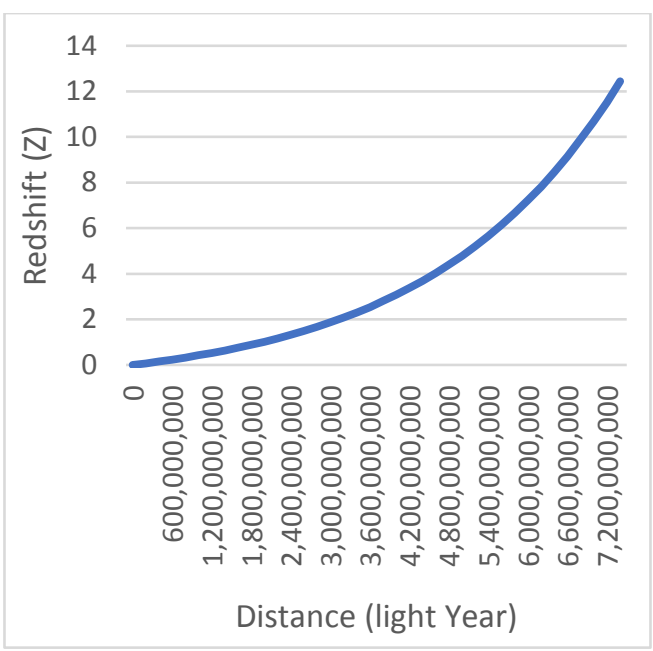

Another thing that we should consider is the value of the $\beta$. Although, the value of the $\mathrm{q}$ is invariant $(q=89875518173474223)$, the value of the $\mathrm{p}$ is not constant. In this paper, we assumed that the parameter $\mathrm{p}$ is the number of quanta masses that each individual $\mathrm{k}$ box of the wave lose in each second and it could be depend on the environmental parameters such as the temperature of the space, hence the obtained distances in the quantum redshift theory should be assumed as the nearest distance of the object. However, we should consider that there is no restriction to decrease one quanta mass from all $\mathrm{k}$ boxes at the same time, $\mathrm{p}$ is the average number of the decreased quanta masses in a second. 
Fig.2 shows a simple model of $\mathrm{k}$ boxes and period of the wave. Fig.3 illustrates a threedimensional model of the virtual $\mathrm{k}$ boxes. Each $\mathrm{k}$ box contains $q=c^{2}+c+1$ quanta masses where $\mathrm{c}=299792458$. Although the capacity of all $\mathrm{k}$ boxes is invariant, $\mathrm{k}$ boxes are not full field at the all-time.

Fig.2: two-dimensional view of an electromagnetic wave and virtual $\mathrm{k}$ box

Fig.2: Three-dimensional view of an electromagnetic wave and virtual $k$ box
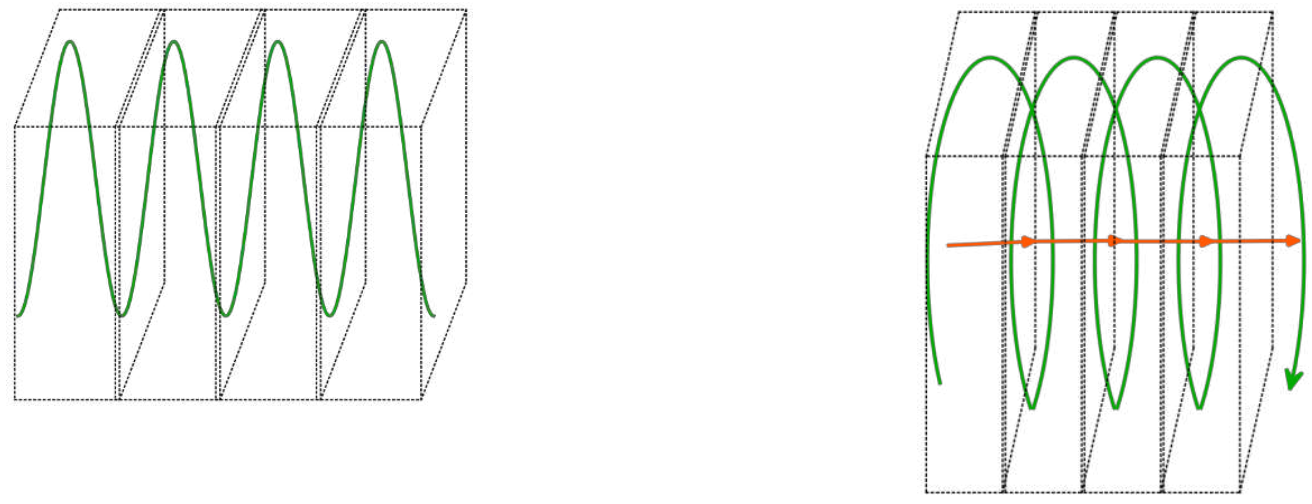

\section{Conclusion}

Quantum redshift predicts distance of the objects in the space. All electromagnetic waves have made of equal number of quanta masses in each period. Losing the quanta masses along the traveling in the space is the reason of decreasing the frequency of the waves.

For the equal z parameters, the distances that obtain by the quantum redshift is less than the same distance in the accelerated expansion space theory. The error of the non-recursive quantum redshift is less than $1 * 10^{-5}$ percent to recursive quantum redshift.

\section{References}

1. Chrisitanson, Gale E. Edwin Hubble: mariner of the nebulae. Routledge, 2019.

2. Slipher, Vesto Melvin. "The radial velocity of the Andromeda Nebula." Lowell Observatory Bulletin 2 (1913): 56-57.

3. Duerbeck, Hilmar W. "Carl Wirtz-An early observational cosmologist." Morphological Cosmology. Springer, Berlin, Heidelberg, 1989. 405-407.

4. Soares, Domingos, and Luiz Paulo R. Vaz. "Solar-Motion Correction in Early Extragalactic Astrophysics." Journal of the Royal Astronomical Society of Canada 108.3 (2014).

5. Kündig, Walter. "Measurement of the transverse doppler effect in an accelerated system." Physical Review 129.6 (1963): 2371.

6. Nezlin, Mikhail V. "Negative-energy waves and the anomalous Doppler effect." UsFiN 120 (1976): 481-495. 
7. Compton, Arthur H., and Ivan A. Getting. "An apparent effect of galactic rotation on the intensity of cosmic rays." Physical Review 47.11 (1935): 817.

8. Byrd, G. G., and M. J. Valtonen. "Origin of redshift differentials in galaxy groups." The Astrophysical Journal 289 (1985): 535-539.

9. Skeivalas, J., V. Turla, and M. Jurevicius. "Predictive models of the galaxies' movement speeds and accelerations of movement on applying the Doppler Effect." Indian Journal of Physics 93.1 (2019): 16.

10. Zhao, HongSheng, John A. Peacock, and Baojiu Li. "Testing gravity theories via transverse Doppler and gravitational redshifts in galaxy clusters." Physical Review D 88.4 (2013): 043013.

11. Albrecht, H-E., et al. Laser Doppler and phase Doppler measurement techniques. Springer Science \& Business Media, 2013.

12. Walker, Jack L. "Range-Doppler imaging of rotating objects." IEEE Transactions on Aerospace and Electronic systems 1 (1980): 23-52.

13. Wolf, Peter, and Gérard Petit. "Satellite test of special relativity using the global positioning system." Physical Review A 56.6 (1997): 4405.

14. Daszkiewicz, M., K. Imilkowska, and J. Kowalski-Glikman. "Velocity of particles in doubly special relativity." Physics Letters A 323.5-6 (2004): 345-350.

15. Delva, P., et al. "Test of special relativity using a fiber network of optical clocks." Physical Review Letters 118.22 (2017): 221102.

16. Tsamparlis, Michael. "Waves in Special Relativity." Special Relativity. Springer, Cham, 2019. 647-702.

17. Ranzan, Conrad. "Cosmic redshift in the nonexpanding cellular universe." American Journal of Astronomy and Astrophysics 2.5 (2014): 47-60.

18. Kaiser, Nick. "Measuring gravitational redshifts in galaxy clusters." Monthly Notices of the Royal Astronomical Society 435.2 (2013): 1278-1286.

19. Müller, Holger, Achim Peters, and Steven Chu. "A precision measurement of the gravitational redshift by the interference of matter waves." Nature 463.7283 (2010): 926-929.

20. Di Dio, Enea, and Uroš Seljak. "The relativistic dipole and gravitational redshift on LSS." Journal of Cosmology and Astroparticle Physics 2019.04 (2019): 050.

21. Delva, Pacôme, et al. "A new test of gravitational redshift using Galileo satellites: The GREAT experiment." Comptes Rendus Physique 20.3 (2019): 176-182.

22. Savalle, Etienne, et al. "Gravitational redshift test with the future ACES mission." Classical and Quantum Gravity 36.24 (2019): 245004.

23. Kalhor, Bahram, and Farzaneh Mehrparvar. "k constant." Available at SSRN 3593865 (2020).

24. Kalhor, Bahram, Farzaneh Mehrparvar, and Behnam Kalhor. "How quantum of the mass, k box, and photon make light and matter?." Available at SSRN 3667603 (2020).

25. Picqué, Nathalie, and Theodor W. Hänsch. "Frequency comb spectroscopy." Nature Photonics 13.3 (2019): 146-157.

26. Gaeta, Alexander L., Michal Lipson, and Tobias J. Kippenberg. "Photonic-chip-based frequency combs." Nature Photonics 13.3 (2019): 158-169.

27. Probst, Rafael A., et al. "A crucial test for astronomical spectrograph calibration with frequency combs." Nature Astronomy (2020): 1-6.

28. Wang, Ning, et al. "Room-temperature heterodyne terahertz detection with quantum-level sensitivity." Nature Astronomy 3.11 (2019): 977-982. 
29. Lara-Avila, S., et al. "Towards quantum-limited coherent detection of terahertz waves in chargeneutral graphene." Nature Astronomy 3.11 (2019): 983-988.

30. Adams, Elizabeth AK, and Joeri van Leeuwen. "Radio surveys now both deep and wide." Nature Astronomy 3.2 (2019): 188-188.

31. Zhao, HongSheng, John A. Peacock, and Baojiu Li. "Testing gravity theories via transverse Doppler and gravitational redshifts in galaxy clusters." Physical Review D 88.4 (2013): 043013. 
Figures

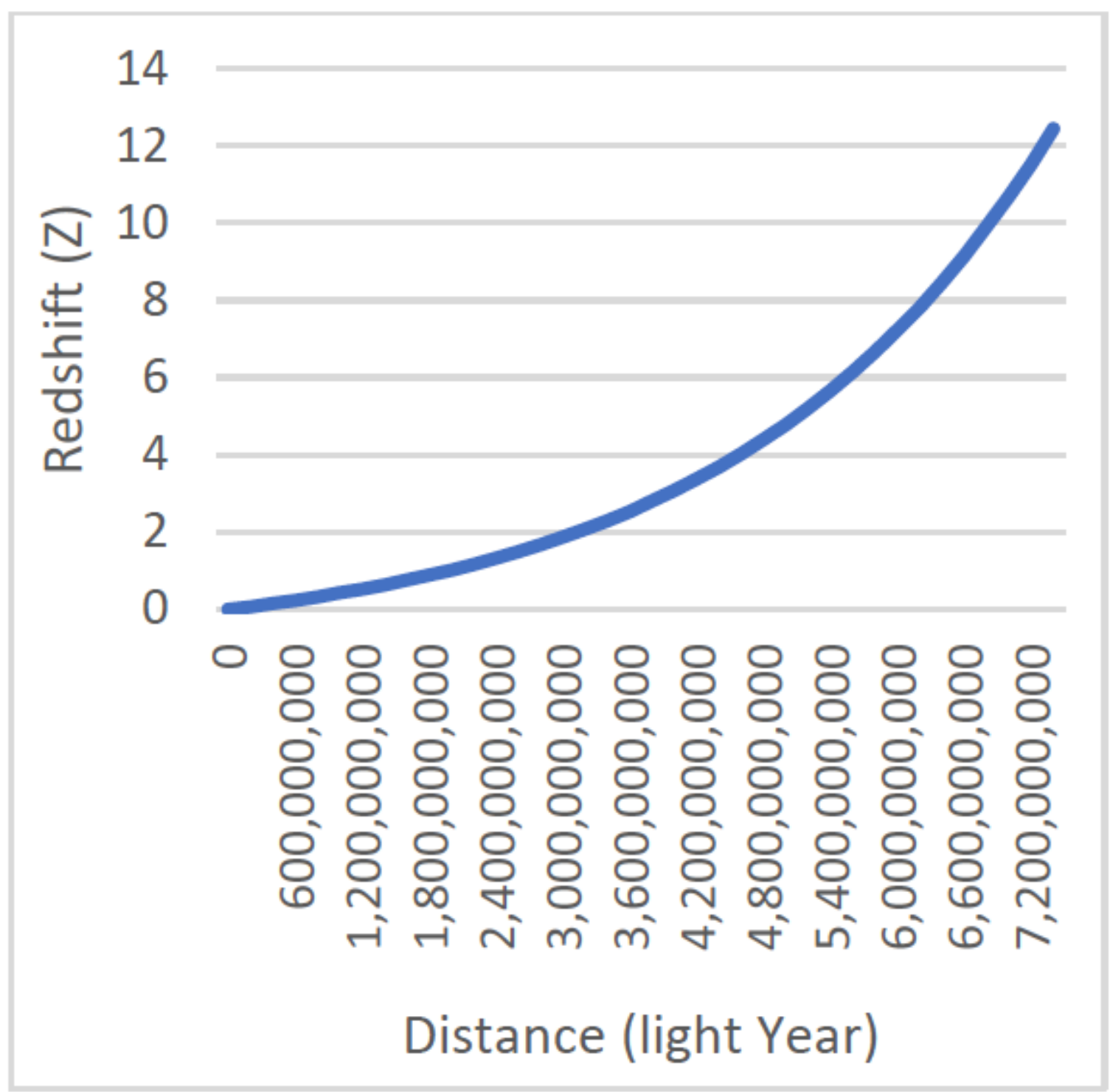

Figure 1

distances and their $\mathrm{z}$ parameters in the quantum redshift 


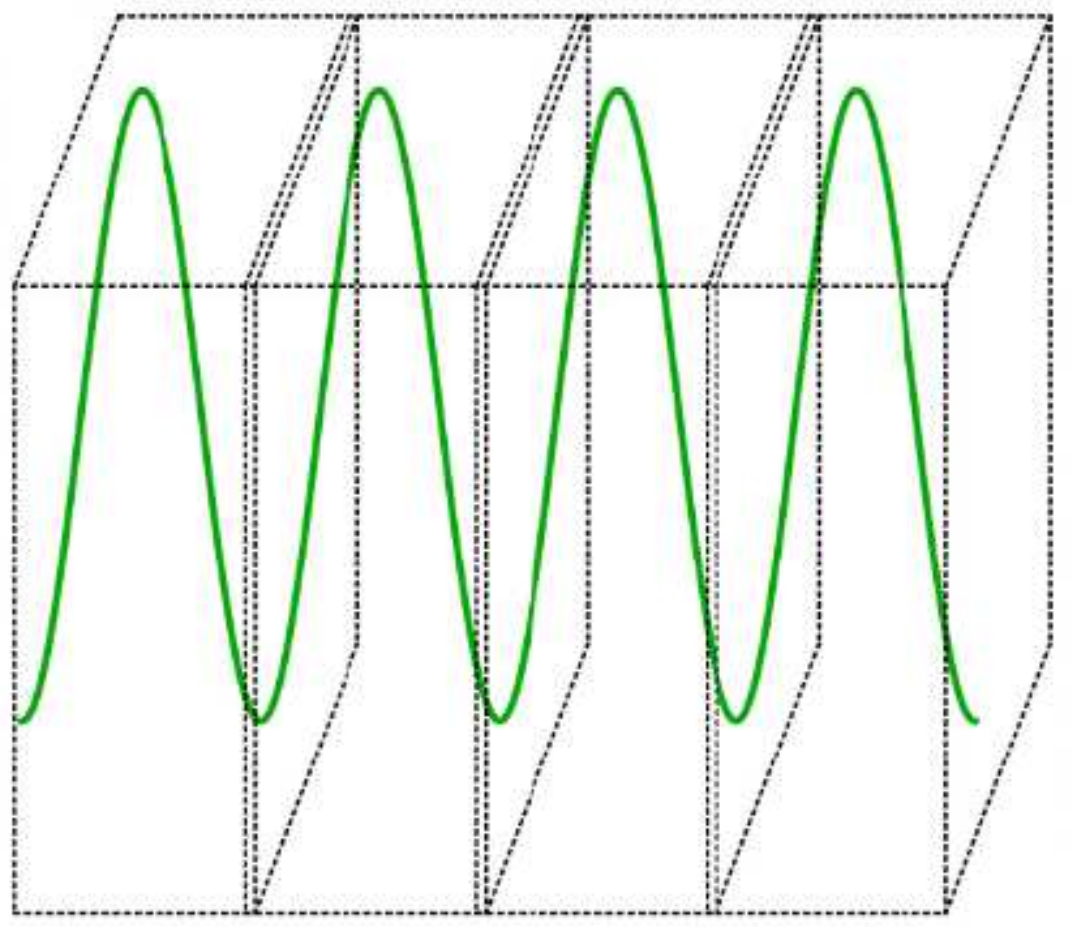

Figure 2

two-dimensional view of an electromagnetic wave and virtual $k$ box 


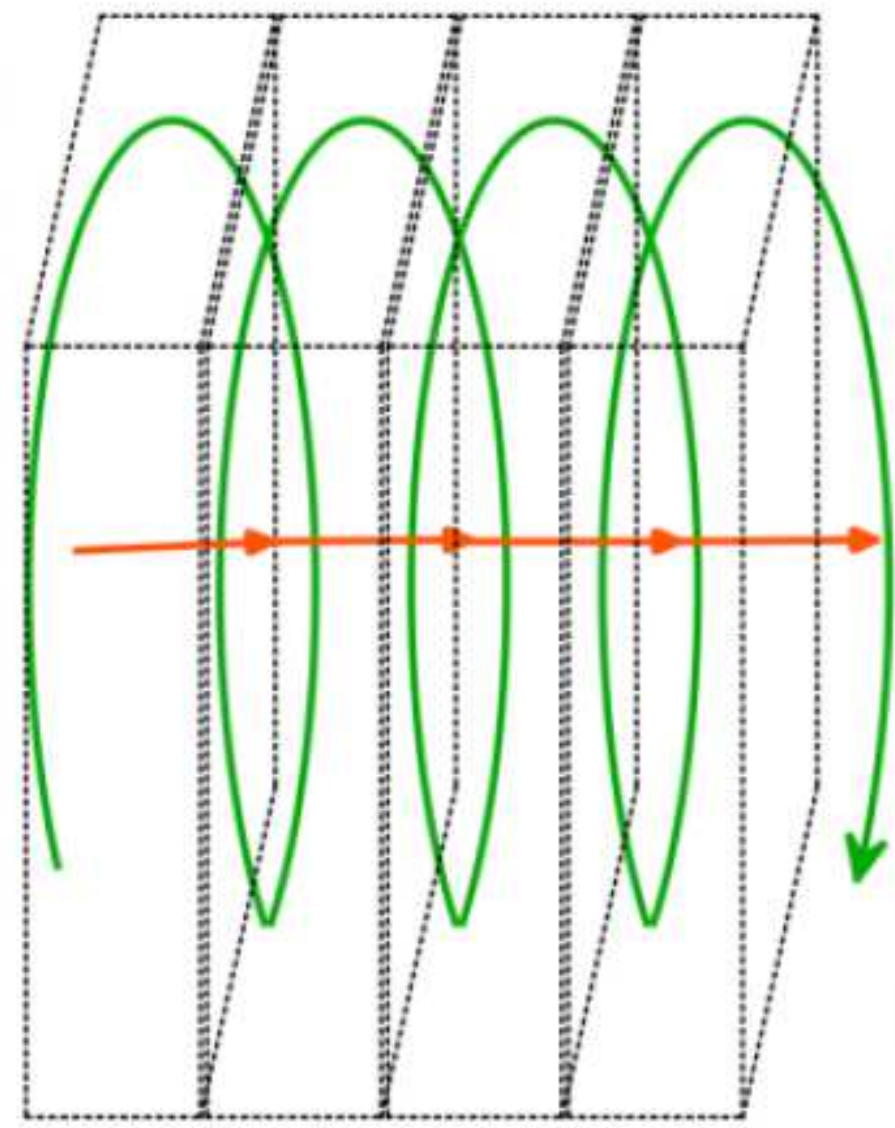

Figure 3

Three-dimensional view of an electromagnetic wave and virtual $k$ box 